\title{
UNIFAC Yöntemi Kullanılarak Etilasetat-Etanol Sistemi İçin Izotermal Buhar-Sıvı Dengesi Kestirimi
}

\author{
Yavuz Salt ${ }^{1 *}$ \\ 1*Yıldız Teknik Üniversitesi, Kimya-Metalürji Fakültesi, Kimya Mühendisliği Bölümü, İstanbul, Türkiye, (ORCID: 0000-0002-1375-6953), salt@ yildiz.edu.tr
} (İlk Geliş Tarihi Aralık 2020 ve Kabul Tarihi Ocak 2021)

(DOI: $10.31590 /$ ejosat.849857)

ATIF/REFERENCE: Salt, Y. (2021). UNIFAC Yöntemi Kullanılarak Etilastat-Etanol Sistemi İçin İzotermal Buhar-Sıvı Dengesi Kestirimi. Avrupa Bilim ve Teknoloji Dergisi, (22), 293-299.

$\ddot{O} \mathbf{z}$

Esterler ve alkollerin endüstriyel ve bilimsel önemi nedeniyle bunların ikili karışımları üzerine çok sayıda araştırma yapılmıştır. Etil asetat, asetik asit ve etanolün Fischer esterleşme reaksiyonuyla üretilebilen önemli bir çözücüdür. Etilasetat saflaştırma işleminde, birkaç ikili ve üçlü azeotroplar oluşmaktadır. Sıvı karışımındaki bileşenlerin aktivite katsayılarının kestiriminin yapılması, buhar-sıvı dengesi (VLE) gibi faz dengesi hesaplamalarında çok önemlidir. Bu nedenle, sıvı karışım özelliklerinin, özellikle aşırı Gibbs serbest enerjisinin $\left(\mathrm{G}^{\mathrm{E}}\right)$ kestirimi için doğru modeller gerekmektedir. Bu çalışmada, etilasetat-etanol sisteminin 298,15 K, 313,15 K ve 323,15 $\mathrm{K}$ sıcaklıklarda izotermal buhar-sıvı denge hesaplamaları gerçekleştirilmiştir. UNIFAC yöntemi kullanılarak aktivite katsayılarının kestirimi yapılmış ve daha sonra aşırı Gibbs serbest enerji değerlerinden ikili etkileşim parametreleri hesaplanmıştır. Sonuçlar, sıcaklığın artışıyla birlikte aktivite katsayılarının ve ikili etkileşim parametrelenin azaldığını göstermiştir. Etil asetat konsantrasyonundaki artışla birlikte aktivite katsayıları azalmış, ikili etkileşim parametreleri artmıştır. Etilasetat-etanol sistemi pozitif aşırı Gibbs serbest enerjisi değerlerine sahiptir ve Raoult yasasından pozitif sapma gösterir.

Anahtar Kelimeler: Buhar-sıvı Dengesi, Etilasetat, Etanol, UNIFAC Yöntemi, İkili Etkileşim Parametresi.

\section{Prediction of Isothermal Vapor-Liquid Equilibrium for the Ethylacetate-Ethanol System Using UNIFAC Method}

\begin{abstract}
Many studies have been conducted on binary mixture of esters and alcohols due to their industrial and scientific importance. Ethyl acetate is an important solvent that can be produced by Fischer esterification reaction of acetic acid and ethanol. In the purification process of ethyl acetate, a few binary and ternary azeotropes are formed. It is crucial to estimate the activity coefficients of components in a liquid mixture for phase equilibrium calculations such as vapor-liquid equilibrium (VLE). Therefore, accurate models are required to estimate liquid mixture properties, particularly excess Gibbs free energy $\left(\mathrm{G}^{\mathrm{E}}\right)$. In the present study, isothermal vapor-liquid equilibrium was calculated for ethyl acetate-ethanol system at various temperatures, i.e., $298.15 \mathrm{~K}, 313.15 \mathrm{~K}$ and 323.15 $\mathrm{K}$. Activity coefficients were estimated using the UNIFAC method, and then binary interaction parameters were calculated from the excess Gibbs free energy values. The results showed that the activity coefficients and binary interaction parameters decreased with the increase in temperature. The activity coefficients decreased as well as binary interaction parameters increased with the increase in ethyl acetate concentration. The ethyl acetate-ethanol system has positive values of the excess Gibbs free energy, and exhibits a positive deviation from Raoult's law.
\end{abstract}

Keywords: Vapor-liquid Equilibrium, Ethylacetate, Ethanol, UNIFAC Method, Binary Interaction Parameter.

\footnotetext{
*Sorumlu Yazar: $\underline{\text { salt @ yildiz.edu.tr }}$
} 


\section{Giriş}

Etil asetat, $\mathrm{CH}_{3} \mathrm{COOC}_{2} \mathrm{H}_{5}$ formülüne sahip ve moleküler ağırlığ 88,10 olan organik bileşiktir. Bu renksiz sıv1, içinde kullanıldığı belirli yapıştırıcılar veya oje çıkarıcılar gibi karakteristik, keskin bir kokuya sahiptir. Etil asetat, etanol ve asetik asitten ester; çözücü olarak kullanılmak üzere büyük ölçekte üretilir. Etil asetat, uçucu, nispeten toksik olmayan ve higroskopik olmayan avantajlara sahip orta derecede polar bir çözücüdür. Zayıf bir hidrojen bağı alıcısıdır ve asidik proton eksikliği nedeniyle donör değildir. Etil asetat\% 3'e kadar suyu çözebilir ve oda sıcaklığında suda \% 8'lik bir çözünürlüğe sahiptir. Güçlü sulu bazlar ve asitlerin varlığında kararsızdır. Alkol, aseton, eter ve kloroform gibi çoğu organik çözücüde çözünür. Ayrıca çevreye ve insana ciddi zarar veren aromatik bileşiklerin yerine konması için birçok kimyasal işlemde çözücü olarak kullanılmıştır (Pattanaik ve Mandalia, 2011).

Birçok bilimsel ve endüstriyel uygulamaya sahip önemli bir endüstriyel kimyasal olan etilasetat endüstriyel verniklerde, matbaa mürekkebinde, boyalarda, tinerlerde ve nitroselüloz verniklerde kullanılan önemli bir çözücüdür ve ayrıca birçok polimerik malzemenin üretimininde de kullanılırlar. Etilasetat saflaştırma işlemi sırasında, ayrılacak birkaç ikili ve üçlü azeotroplar yapılanmaktadır (Zhang ve ark., 2009; Ghanadzadeh ve ark., 2012; Xia ve ark., 2012). Etil asetat üretiminde asetik asidin Fischer esterleştirme reaksiyonu ve etanol dehidrojenasyon yöntemi kullanılmaktadır. $\mathrm{Bu}$ işlemlerde, etil asetat ve etanol karışımı atmosfer basıncında geleneksel distilasyon ile ayrilması zor olan bir minimum azeotrop oluşturur (Zhang ve ark., 2008; Zhang ve ark., 2009; Zhang ve ark., 2017). Çözücü karışımları, eczacılık ve kozmetikte, suda az çözünür aktif bileşenlerin çözünürlüğünü arttırmak için yaygın olarak kullanılmaktadır. 1,2-propandiol ve etanol, ilaç tasarım işlemlerinde en çok kullanılan yardımcı çözücülerdir. Etil asetat, olası tüm bileşimlerde etanol ile karışabilen yaygın bir organik çözücüdür (Ruidiaz ve ark., 2010).

Kimyasal proses tasarımı genellikle sıvı karışımların ayrılması ile ilgilidir ve çoğunlukla sıvı faz aktivite katsayılarının belirlenmesi gerekmektedir. Sıvı karışımların termodinamik özelliklerinin belirlenmesi, distilasyon, ekstraksiyon veya absorpsiyon kolonları gibi kimyasal üretim ekipmanlarının tasarımında önemli bir konu durumundadır. $\mathrm{Bu}$ nedenle, kimyasal proses simülasyonunda sıvı karışım özelliklerini, özellikle aktivite katsayısı verilerini kullanarak aşırı Gibbs enerjisini $\left(\mathrm{g}^{\mathrm{E}}\right)$ kestirimi için doğru bir modele ihtiyaç duyulmaktadır. (Fredenslund ve ark., 1975; Huang ve Lee, 1996; Parsana ve Parikh, 2015). Özellikle deneysel verilerin mevcut olmadığı durumlarda mevcut verilerden yola çıkarak faz dengesi verilerinin kestirimin yapılmasında birçok grup katkısı yöntemi kullanılmaktadır. Bu yöntemlerdeki temel fikir, binlerce kimyasal bileşik olmasına rağmen bu bileşikleri oluşturan fonksiyonel grupların sayısının az olmasina ve bir sıvının fiziksel bir özelliğinin molekülün fonksiyonel grupları tarafından yapılan katkıların toplamı olduğu varsayımına dayanmaktadır. Bu varsayım; bir grubun katkısının başka bir grubun katkısından bağımsız olduğunu ve bir moleküldeki herhangi bir grubun etkisinin, o molekül içindeki diğer grupların doğasından etkilenmediğini ifade etmektedir (Fredenslund ve ark., 1975).

Önemli grup katkı yöntemleri arasında ASOG (Analytical Solution of Groups-Grupların Analitik Çözümü) ve UNIFAC (Uniquac Functional Group Activity Coefficient-Uniquac Grup Aktivite Katsayısı) yöntemleri öne çıkmaktadır (Sandler, 2017).
UNIFAC yöntemi, düşük ve orta basınçlarda ve 300 ile $425 \mathrm{~K}$ arasındaki sıcaklıklarda, elektrolit olmayan, polimerik olmayan karışımlarda termokimyasal özelliklerin belirlenmesi ve sıvı faz aktivite katsayılarının kestirimi için güvenilir ve hızlı bir yöntemdir. (Gmehling, ve ark., 1982; Letyanina ve ark., 2016). Ayrıca, farklı moleküller arasındaki çekim kuvvetlerini değerlendirebilmek için etkileşim parametrelerinin belirlenmesi gerekmektedir (Kobuchi ve ark., 2013). Kavram olarak, UNIFAC yöntemi, karışımlardaki aktivite katsayılarının yapısal gruplar arasındaki etkileşimlerle ilişkili olduğu ASOG yöntemini takip eder (Poling ve ark., 2001).

Moleküler aktivite katsayısı iki kısma ayrılır: bir kısım moleküler boyut ve şekil farklılıkları nedeniyle katkı sağlayan tümleşik (combinatorial) terim, diğeri moleküler etkileşimler nedeniyle katkıyı sağlayan artık (residual) terim. Aktivite katsayısına artık katkı grup alanları ve grup etkileşimlerine bağlıdır. UNIQUAC yönteminde tümleşik terim boyut parametreleri için grup katkılarını kullanırken artık terim her bir ikili sisteme ait iki ayarlanabilir parametre içerir. UNIFAC yönteminde her iki katkı terimi de grup katkıları kullanılarak bulunmaktadır. Böylelikle karışımın moleküllerden değil fonksiyonel gruplardan oluştuğu düşünülmektedir. UNIFAC yönteminde UNIQUAC aktivite katsayılarının tümleşik kısmı doğrudan kullanılmakta ve bu kısmı sadece saf bileşen özellikleri kullanılmaktadır (Poling ve ark., 2001; Sandler, 2017).

Etil asetat-etanol sisteminin buhar-sıv1 denge verilerinin elde edilmesine yönelik literatürde farklı çalışmalar yapılmıştır. Furnas ve Leighton (1937) tarafindan sunulan çalışmada atmosferik basınçta etanol-etil asetat ve etil asetat-asetik asit sistemi için buhar-sıvı denge verilerini vermişlerdir. Murti ve Van Winkle (1957), etilasetatın methanol, etanol, 1-propanol ve 2-propanol ile ikili karışımları ve 1-propanol-su sistemi için 760 $\mathrm{mmHg}$ basınçlık izobarik koşullar için buhar sıvı denge verilerini elde etmişler ve 40 ve $60^{\circ} \mathrm{C}$ için toplam basınç değişimini ortaya koymuşlardır. Ortega ve ark. (1986), 760 mmHg'lık izobarik koşullarda etilasetat-etanol karışımlarının buhar-sıvı dengesini çalışmışlar ve deneysel verileri elde ederek aktivite katsayılarını hesaplamışlardır. İzotermal koşullar altında birçok farklı ikili karışım için de olmak üzere Nagata ve ark., (1975) etilasetat/etanol sisteminin $55^{\circ} \mathrm{C}$ sicaklıkta deneysel olarak buhar-sıvı denge verilerini elde etmişler ve Wilson denklemini kullanarak aşırı Gibbs enerjilerini hesaplamışlardır. Diğer bir çalışmada, Susial ve ark. (2011), 0,1, 0,5 ve 0,7 MPa basınçlarında olmak üzere izobarik VLE verilerini deneysel bir çalışmayla belirlemiş ve UNIFAC ve ASOG yönteminin kestirim doğruluğunu incelemişlerdir. Ayrıca, Kobuchi ve ark. (2013), Wilson parametrelerinin hesaplanmasına yönelik olarak, saf bileşen için önerilen bir yöntemle ester içerikli ikili karışımların etkileşim parametrelerini sunmuşlar ve etil asetat-etanol ve Ipropil asetat-2-propanol sistemleri için 101,3 kPa basınçta VLE verilerini karşılaştırmışlardır.

$\mathrm{Bu}$ çalışmada, etilasetat-etanol sisteminin 298,15K, $313,15 \mathrm{~K}$ ve $323,15 \mathrm{~K}$ sicaklıklarda izotermal buhar-sıvı denge hesaplamaları gerçekleştirilmiştir. Çalışma kapsamında hazırlanan Excel programına literatürde verilen parametreler ve ilgili eşitlikler girilerek oluşturulan hesaplama prosedürü çerçevesinde UNIFAC yöntemi kullanılarak aktivite katsayılarının kestirimi yapılmış ve aşırı Gibbs (fark) enerjileri bulunarak ikili etkileşim parametreleri hesaplanmıştır. 


\section{Materyal ve Metot}

\subsection{Materyal}

Etilasetat ve etanolün bazı özellikleri Tablo 1'de verilmiştir.

Tablo 1. Etilasetat ve Etanolün Bazı Fiziksel ve Kimyasal Özellikleri (Abraham ve ark., 1971; Barton, 1975; Khasanshin, 1984; Mulder and Smolders, 1986; Nijhius ve ark., 1993; Dean, 1999; Resa ve ark., 2004; Mackay ve ark., 2006; Ruidiaz ve ark., 2010).

\begin{tabular}{|c|c|c|c|c|c|c|}
\hline Özellik & \multicolumn{3}{|c|}{ Etilasetat } & \multicolumn{3}{|c|}{ Etanol } \\
\hline M.A. & \multicolumn{3}{|c|}{$88,10 \mathrm{~g} / \mathrm{mol}$} & \multicolumn{3}{|c|}{$46,07 \mathrm{~g} / \mathrm{mol}$} \\
\hline$T_{\text {kaynama }}$ & \multicolumn{3}{|c|}{$77,11^{\circ} \mathrm{C}$} & \multicolumn{3}{|c|}{$78,29^{\circ} \mathrm{C}$} \\
\hline$T_{c}$ & \multicolumn{3}{|c|}{$250,2^{\circ} \mathrm{C}$} & \multicolumn{3}{|c|}{$240,9^{\circ} \mathrm{C}$} \\
\hline$P_{c}$ & \multicolumn{3}{|c|}{$38,31 \mathrm{~atm}$} & \multicolumn{3}{|c|}{$60,57 \mathrm{~atm}$} \\
\hline$V_{c}$ & \multicolumn{3}{|c|}{$286 \mathrm{~cm}^{3} / \mathrm{mol}$} & \multicolumn{3}{|c|}{$167 \mathrm{~cm}^{3} / \mathrm{mol}$} \\
\hline$\rho_{c}$ & \multicolumn{3}{|c|}{$0,378 \mathrm{~g} / \mathrm{cm}^{3}$} & \multicolumn{3}{|c|}{$0,276 \mathrm{~g} / \mathrm{cm}^{3}$} \\
\hline \multirow[t]{2}{*}{$\delta^{*}$} & \multicolumn{3}{|c|}{$8,9 \mathrm{cal}^{1 / 2} \mathrm{~cm}^{-3 / 2}$} & \multicolumn{3}{|c|}{$13,0 \mathrm{cal}^{1 / 2} \mathrm{~cm}^{-3 / 2}$} \\
\hline & $298,15 K$ & $313,15 K$ & $323,15 K$ & $298,15 K$ & $313,15 K$ & $323,15 K$ \\
\hline$V^{* *}$ & 98,55 & 100,69 & 102.1 & 58,69 & 59,68 & 60,38 \\
\hline$\rho$ & 0,894 & 0,875 & 0,863 & 0,785 & 0,772 & 0,763 \\
\hline
\end{tabular}

\subsection{Materyal}

\subsubsection{Ikili Etkileşim Parametresi ve Aktivite Katsayısı}

Flory-Huggins teorisine göre, bileşen $1(\mathrm{EtAc})$ ve $2(\mathrm{EtOH})$ arasındaki ikili etkileşim parametresi şu şekilde verilmektedir (Mulder ve ark., 1985; Rhim and Huang, 1989):

$$
\chi_{12}=\frac{1}{x_{1} v_{2}}\left[x_{1} \ln \left(x_{1} / v_{1}\right)+x_{2} \ln \left(x_{2} / v_{2}\right)+G^{E} / R T\right]
$$

$\mathrm{Bu}$ eşitlikte; $\mathrm{x}$ ve $\mathrm{v}$ sırasıyla mol kesri ve hacim kesridir. Aşırı Gibbs enerjileri izotermal buhar-sıvı denge verilerinden yararlanarak aşağıda verilen eşitlikten bulunabilir (Nagata ve ark., 1975; Letyanina ve ark., 2016):

$$
\mathrm{G}^{\mathrm{E}}=\mathrm{RT} \sum_{\mathrm{i}} \mathrm{x}_{\mathrm{i}} \ln \gamma_{\mathrm{i}}
$$

Aktivite katsayıları $\left(\gamma_{\mathrm{i}}\right)$ ise UNIFAC yöntemi kullanılarak hesaplanabilmektedir (Poling ve ark., 2001). UNIFAC yönteminde aktivite katsayısı iki kısımdan oluşmaktadır: tümleşik ve artık.

$$
\ln \gamma_{i}=\ln \gamma_{i}^{C}+\ln \gamma_{i}^{R}
$$

Aktivite katsayısına moleküllerin büyüklük ve şekliyle ilgili olan tümleşik katkı aşağıdaki eşitlikle verilmektedir:

$$
\ln \gamma_{\mathrm{i}}^{\mathrm{C}}=\ln \frac{\Phi_{\mathrm{i}}}{\mathrm{x}_{\mathrm{i}}}+\frac{\mathrm{z}}{2} \mathrm{q}_{\mathrm{i}} \ln \frac{\theta_{\mathrm{i}}}{\Phi_{\mathrm{i}}}+\mathrm{l}_{\mathrm{i}} \frac{\Phi_{\mathrm{i}}}{\mathrm{x}_{\mathrm{i}}} \sum_{\mathrm{j}} \mathrm{x}_{\mathrm{j}} 1_{\mathrm{j}}
$$

$\theta_{\mathrm{i}}$ and $\Phi_{\mathrm{i}}$ aşağıdaki eşitliklerle verilen alan ve segment kesirleridir:

$$
\theta_{\mathrm{i}}=\frac{\mathrm{q}_{\mathrm{i}} \mathrm{x}_{\mathrm{i}}}{\sum_{\mathrm{j}} \mathrm{q}_{\mathrm{j}} \mathrm{x}_{\mathrm{j}}}
$$

$$
\begin{aligned}
& \Phi_{\mathrm{i}}=\frac{\mathrm{r}_{\mathrm{i}} \mathrm{x}_{\mathrm{i}}}{\sum_{\mathrm{j}} \mathrm{r}_{\mathrm{j}} \mathrm{x}_{\mathrm{j}}} \\
& \mathrm{l}_{\mathrm{i}}=\frac{\mathrm{z}}{2}\left(\mathrm{r}_{\mathrm{i}}-\mathrm{q}_{\mathrm{i}}\right)-\left(\mathrm{r}_{\mathrm{i}}-1\right)
\end{aligned}
$$

Koordinasyon sayısı z, 10'a eşittir. $r_{i}$ ve $q_{i}$ molar grup hacim ve grup alan parametrelerinden hesaplanabilir:

$$
\begin{aligned}
& \mathrm{r}_{\mathrm{i}}=\sum_{\mathrm{k}} \mathrm{v}_{\mathrm{k}}^{(\mathrm{i})} \mathrm{R}_{\mathrm{k}} \\
& \mathrm{q}_{\mathrm{i}}=\sum_{\mathrm{k}} \mathrm{v}_{\mathrm{k}}^{(\mathrm{i})} \mathrm{Q}_{\mathrm{k}}
\end{aligned}
$$

Burada $v_{\mathrm{k}}^{(\mathrm{i})} i$ molekülündeki $k$ tipli grupların sayısıdır. Residual kısım ise grup alanlarına ve grup etkileşimlerine bağlıdır:

$$
\ln \gamma_{\mathrm{i}}^{\mathrm{R}}=\sum_{\mathrm{k}} \mathrm{v}_{\mathrm{k}}^{(\mathrm{i})}\left(\ln \Gamma_{\mathrm{k}}-\ln \Gamma_{\mathrm{k}}^{(\mathrm{i})}\right)
$$

$\Gamma_{\mathrm{k}}$, grup residual aktivite katsay1s1 ve $\Gamma_{\mathrm{k}}^{(\mathrm{i})}$ sadece $i$ tipli moleküller için $k$ grubunun resiual aktivite katsayısıdır. Grup aktivite katsayısı aşağıdaki denklem kullanılarak hesaplanabilir:

$$
\begin{aligned}
& \ln \Gamma_{\mathrm{k}}=\mathrm{Q}_{\mathrm{k}}\left[1-\ln \left(\sum_{\mathrm{m}} \theta_{\mathrm{m}} \psi_{\mathrm{mk}}\right)-\sum_{\mathrm{m}} \frac{\theta_{\mathrm{m}} \psi_{\mathrm{km}}}{\sum_{\mathrm{n}} \theta_{\mathrm{n}} \psi_{\mathrm{nm}}}\right] \\
& \theta_{\mathrm{m}}=\frac{\mathrm{q}_{\mathrm{m}} \mathrm{X}_{\mathrm{m}}}{\sum_{\mathrm{n}} \mathrm{q}_{\mathrm{n}} \mathrm{X}_{\mathrm{n}}}
\end{aligned}
$$

Burada $\theta_{\mathrm{m}} m$ grubunun alan kesri ve $\mathrm{X}_{\mathrm{m}}$ karışımdaki $m$ grubunun mol kesridir. Grup etkileşim parametresi sıcaklığa bağımlı olarak aşağıdaki eşitlikten bulunabilir:

$$
\psi_{\mathrm{mn}}=\exp \left(-\frac{\mathrm{a}_{\mathrm{mn}}}{\mathrm{T}}\right)
$$

$\mathrm{a}_{\mathrm{mn}}$ grup etkileşim parametresidir $\left(\mathrm{a}_{\mathrm{mn}} \neq \mathrm{a}_{\mathrm{nm}}\right)$. Ayrıca, $\mathrm{a}_{1,1}$, $\psi_{5,5}$ and $\psi_{11,11}=0,0$ olduğundan dolay $1 \psi_{1,1}=\psi_{5,5}=\psi_{11,11}=$ 1,0 olarak alınmalıdır.

\subsubsection{Buhar-sıvı Denge Hesaplamast}

Buhar evresinin ideal gaz olarak kabul edilebileceği kadar düşük basınçlarda buhar evresi bileşimi ve toplam basınç (P) iki bileşenli bir karışım için aşağıdaki eşitliklerden hesaplanabilir (Kobuchi ve ark., 2013):

$$
\begin{aligned}
& \mathrm{y}_{\mathrm{i}} \mathrm{P}=\mathrm{P}_{\mathrm{i}}^{\mathrm{S}} \mathrm{x}_{\mathrm{i}} \gamma_{\mathrm{i}} \quad \mathrm{i}=1,2 \\
& \mathrm{P}=\mathrm{P}_{1}^{\mathrm{S}} \mathrm{x}_{1} \gamma_{1}+\mathrm{P}_{2}^{\mathrm{S}} \mathrm{x}_{2} \gamma_{2}
\end{aligned}
$$

$\mathrm{P}_{1}^{\mathrm{s}}$ ve $\mathrm{P}_{2}^{\mathrm{s}}$ sırasıyla etilasetat ve etanolün buhar basınçlarıdır ve Antoine eşitliği kullanılarak hesaplanabilir (Poling et al., 2001): 


$$
\log \left(\mathrm{P}_{\mathrm{i}}^{\mathrm{s}} / \mathrm{bar}\right)=\mathrm{A}-\frac{\mathrm{B}}{\mathrm{T}+\mathrm{C}-273,15}
$$

Tablo 2. Etilasetat ve Etanol için Antoine Sabitleri (Poling et al., 2001)

\begin{tabular}{lccc} 
& \multicolumn{3}{c}{ Antoine Sabitleri } \\
\cline { 2 - 4 } Bileșen & A & B & C \\
\hline Etilasetat & 4,13361 & 1195,13 & 212,47 \\
Etanol & 5,33675 & 1648,22 & 230,918 \\
\hline
\end{tabular}

Etilasetat-etanol ikili sistemi için sıcaklık (T) ve etilasetat sıvı evresi mol kesri $\left(\mathrm{x}_{1}\right)$ girdileri kullanılarak buhar-sıvı denge hesaplamaları gerçekleştirilmiştir. İlk olarak UNIFAC grup katkı yöntemiyle sırasıyla etilasetat ve etanol için olmak üzere $\gamma_{1}$ ve $\gamma_{2}$ aktivite katsayılarının kestirimi yapılmıştır. Daha sonra Antoine eşitliğinden buhar basınçları bulunarak toplam basınç bulunmuş ve buhar-sıvı dengesi eşitliğinden buhar evresi bileşimi hesaplanmıştır.

\section{Araştırma Sonuçları ve Tartışma}

EtOAc(1)-EtOH(2) sistemi için UNIFAC grup spesifikasyonları ve parametreleri Tablo 3'de, hesaplanan grup ve grup-grup etkileşimleri ise Tablo 4'de verilmiştir. Aşırı Gibbs fonksiyonları, aktivite katsayıları ve ikili etkileşim parametresi değerleri EtOAc(1)-EtOH(2) sistemi için karışım bileşiminin fonksiyonu olarak 298,15 K, 313,15 K ve $323,15 \mathrm{~K}$ sıcaklıklarında hesaplanmıştır. Elde edilen sonuçlar Tablo 5 ve 6'da verilmiştir.

Etilasetat sıvı mol kesriyle aktivite katsayılarının, aşırı Gibbs enerjisinin ve buhar mol kesrinin değişimi sırasıyla Şekil 1 ve 2'de, etilasetat sivi ve buhar mol kesirlerinin toplam basınçla değişimi ise sırasıyla Şekil 3 ve 4'de gösterilmiştir. Pozitif Gibbs enerjisi değerlerine sahip EtOAc (1)-EtOH (2) sisteminin Raoult yasasından pozitif sapma sergilediği açıtır. Susial ve ark. (2011), görülen bu pozitif sapmanın alkoldeki hidrojen bağlanmasından ve ester grubundaki $\pi$ - elektronlarıyla dipolar kuvvetten kaynaklanabileceğini belirtmiştir.

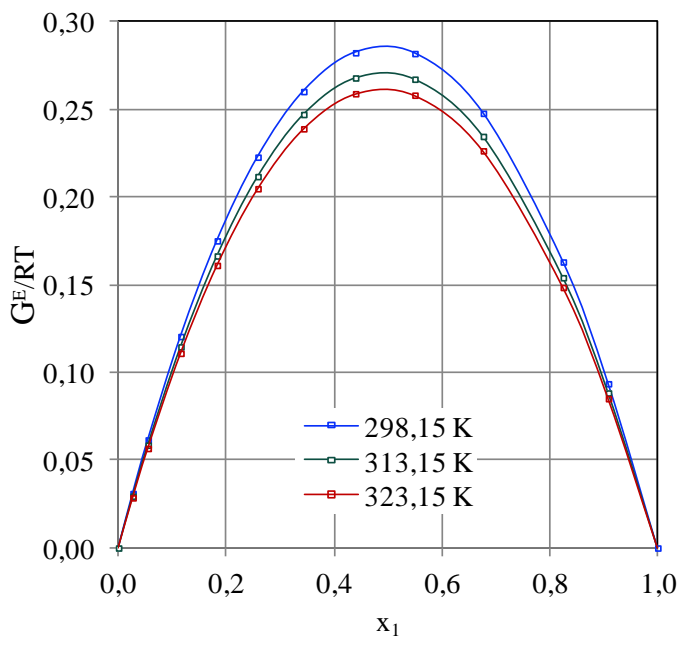

Şekil 1. EtOAc(1)-EtOH(2) Sistemi için Farklı Sıcaklıklarda Aşırı Gibbs Enerjisinin Değişimi

1'den büyük aktivite katsayıları, iki bileşen arasındaki çekim kuvvetlerinin zayıf olduğunu göstermektedir. Ayrıca aşırı
Gibbs enerjisi üzerinde bileşimin önemli bir etkiye sahip olduğu görülmektedir. Anila ve ark. (2016) bunun benzer olmayan moleküller arasındaki dağılım kuvvetlerinden kaynaklanabileceğini bildirmiştir. Ayrıca, ikili etkileşim parametresi, karışımdaki etil asetat konsantrasyonu artışıyla birlikte artmakta, sıcaklık artışılla birlikte ise azalmaktadır.

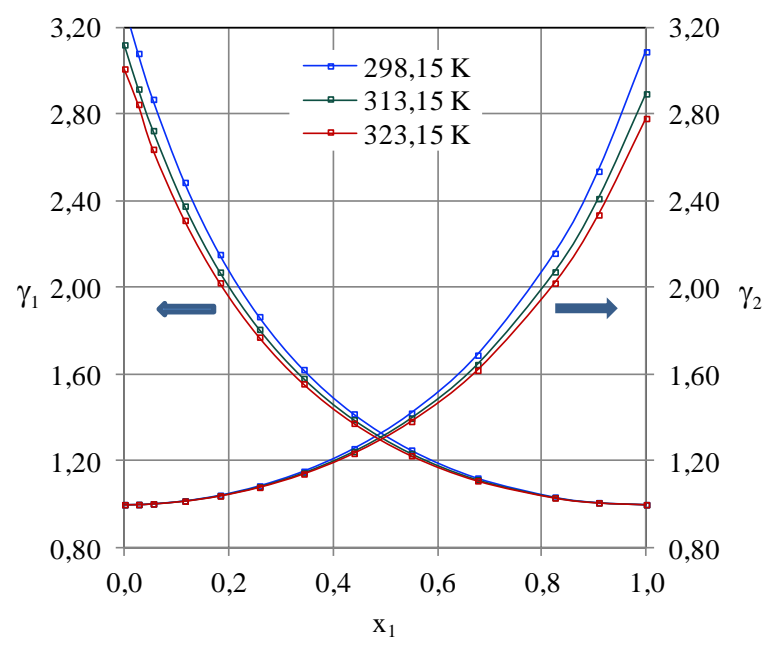

Şekil 2. EtOAc(1)-EtOH(2) Sistemi için Farklı Sıcaklıklarda Aktivite Katsayılarının Değişimi

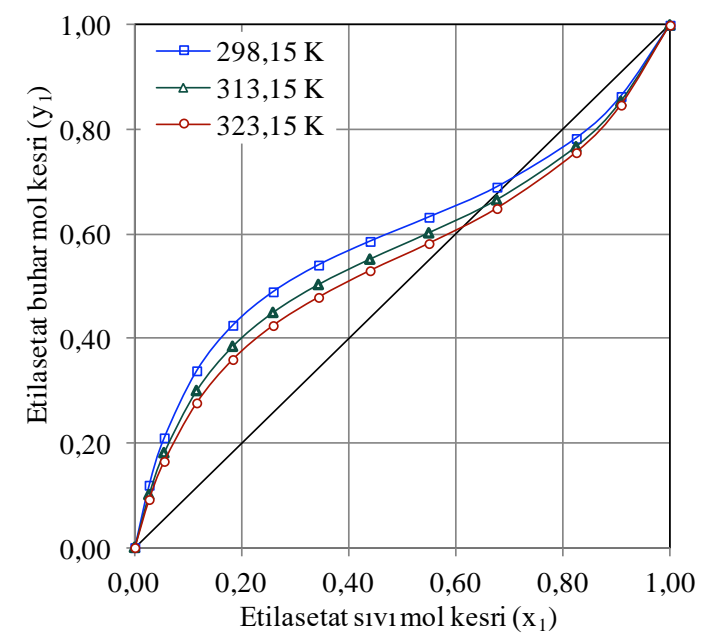

Şekil 3. EtOAc(1)-EtOH(2) Sistemi için Sıvı Mol Kesriyle Buhar Mol Kesri Değişimi

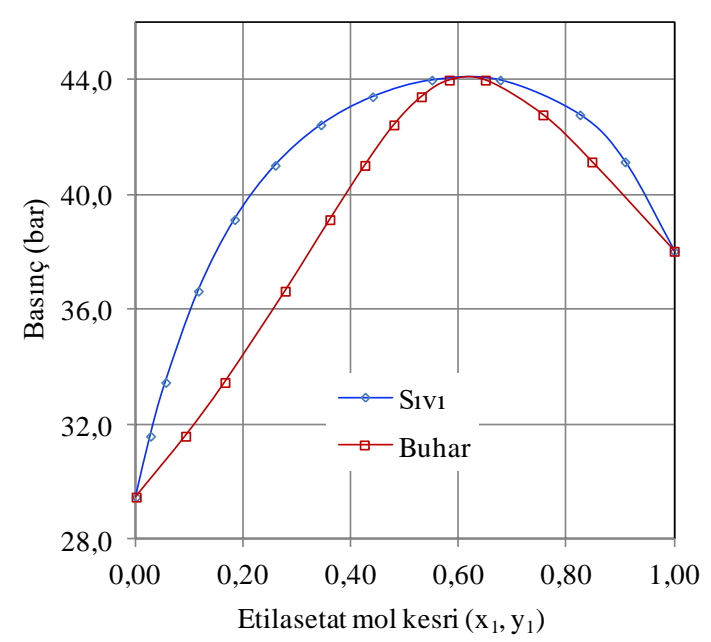

Şekil 4. EtOAc(1)-EtOH(2) Sistemi için Mol Kesriyle Toplam Basınç Değişimi 
Tablo 3. EtOAc(1)-EtOH(2) Sistemi için UNIFAC Grup Spesifikasyonlart ve Parametreleri (Poling et al., 2001)

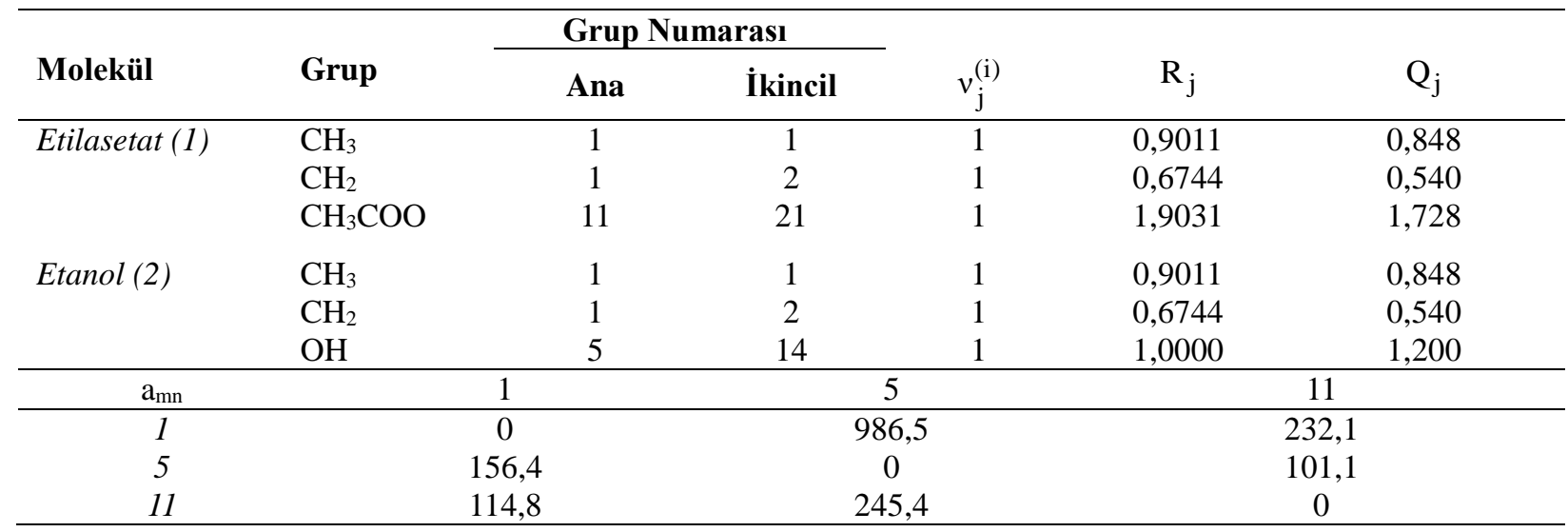

Tablo 4. EtOAc(1)-EtOH(2) Sistemi için Hesaplanan Grup-Grup Etkileşimleri

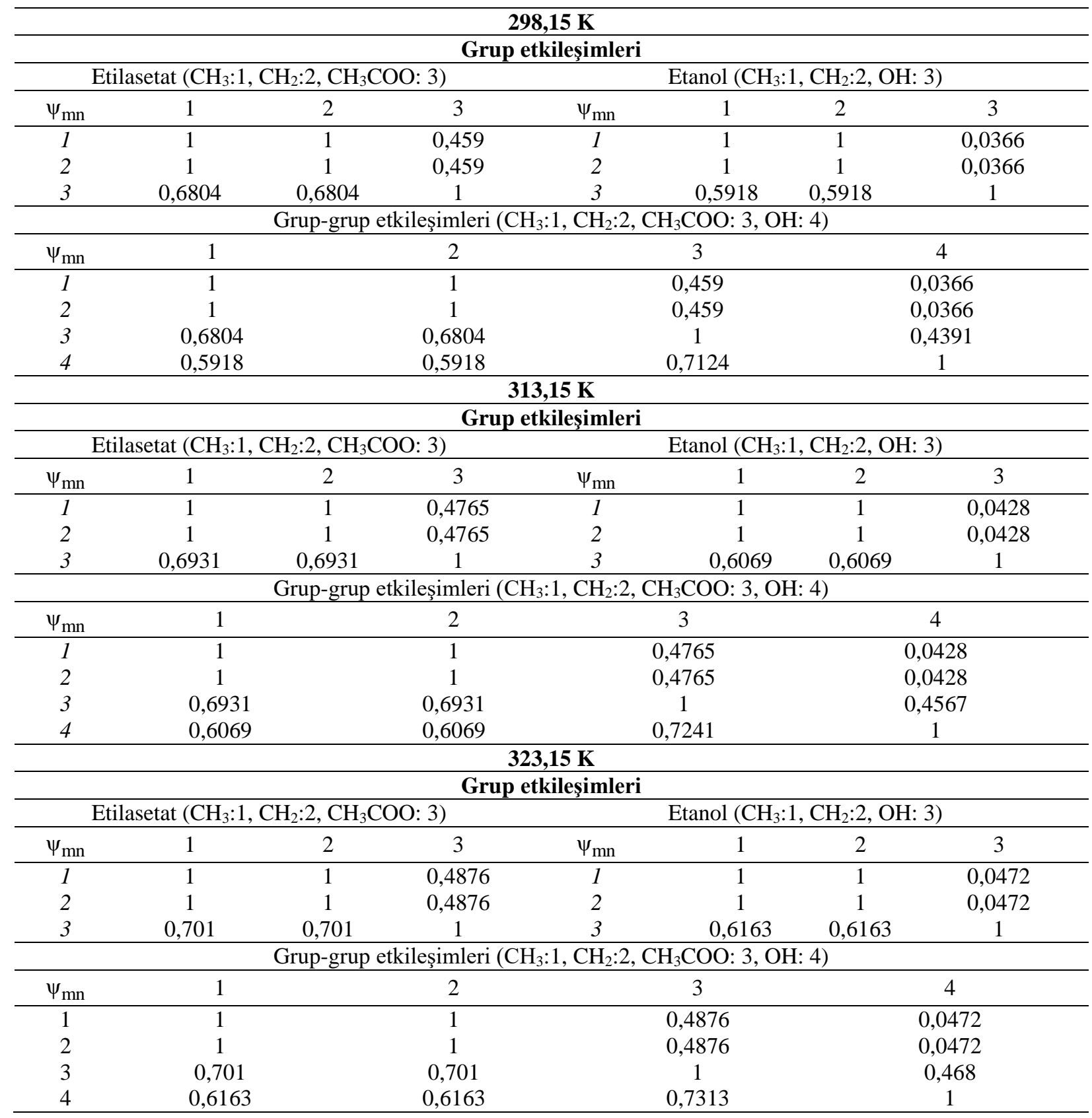


Avrupa Bilim ve Teknoloji Dergisi

Tablo 5. EtOA (1)-EtOH (2) Sistemi için Farklı Stcakliklarda (T, K) Buhar-Sıvı Denge Verileri ve Aktivite Katsayıları

\begin{tabular}{|c|c|c|c|c|c|c|c|c|c|c|c|c|}
\hline \multirow{2}{*}{$x_{1}$} & \multicolumn{3}{|c|}{$\mathbf{y}_{1}$} & \multicolumn{3}{|c|}{$\mathbf{P}(\mathbf{k P a})$} & \multicolumn{3}{|c|}{$\gamma_{1}$} & \multicolumn{3}{|c|}{$\gamma_{2}$} \\
\hline & 298,15 & 313,15 & 323,15 & 298,15 & 313,15 & 323,15 & 298,15 & 313,15 & 323,15 & 298,15 & 313,15 & 323,15 \\
\hline 0,027 & 0,119 & 0,101 & 0,092 & 8,71 & 19,40 & 31,60 & 3,0807 & 2,9165 & 2,8457 & 1,0010 & 1,0009 & 1,0006 \\
\hline 0,055 & 0,210 & 0,181 & 0,165 & 9,46 & 20,74 & 33,47 & 2,8690 & 2,7250 & 2,6387 & 1,0040 & 1,0038 & 1,0037 \\
\hline 0,116 & 0,338 & 0,300 & 0,277 & 10,71 & 23,00 & 36,65 & 2,4862 & 2,3768 & 2,3109 & 1,0175 & 1,0167 & 1,0162 \\
\hline 0,183 & 0,426 & 0,385 & 0,360 & 11,69 & 24,76 & 39,14 & 2,1536 & 2,0723 & 2,0230 & 1,0434 & 1,0414 & 1,0401 \\
\hline 0,259 & 0,490 & 0,450 & 0,425 & ,43 & 5,11 & 41 , & 1,8671 & 1,8083 & 1,7726 & 1,0864 & 1,0823 & 1,0798 \\
\hline 0,343 & 0,541 & 0,503 & 0,479 & 13,00 & 27,12 & 42,44 & 1,6230 & 1,5822 & 1,5572 & 1,1539 & 1,1463 & 1,1416 \\
\hline 0,440 & 0,586 & 0,552 & 0,530 & 13,42 & 27,85 & 43,42 & 1,4185 & 1,3917 & 1,3753 & 1,2582 & 1,2449 & 1,2365 \\
\hline 0,550 & 0,632 & 0,602 & 0,5 & & & & & 1,2358 & 1,2 & 219 & 1,3983 & 1,3836 \\
\hline 0,677 & 0,690 & 0,665 & 0,6 & & 28,47 & & 27 & 1,1148 & 1,1099 & 1,6894 & 1,6461 & 1,6195 \\
\hline 0,825 & 0,783 & 0,767 & 0,756 & 13,75 & 27,89 & 42,79 & 1,0348 & 1,0325 & 1,0310 & 2,1593 & 2,0735 & 2,0218 \\
\hline 0,909 & 0,864 & 0,854 & 0,847 & 13,40 & 26,96 & 41,15 & 1,0094 & 1,0088 & 1,0084 & 2,5374 & 2,4112 & 2,3360 \\
\hline
\end{tabular}

Tablo 6. EtOAc (1)-EtOH (2) Sistemi için Aşırı Gibbs Enerjileri ve İkili Etkileşim Parametresi Değerleri

\begin{tabular}{ccccccc}
\hline \multirow{2}{*}{$\mathbf{x}_{\mathbf{1}}$} & \multicolumn{3}{c}{$\mathrm{G}^{\mathrm{E}}(\mathbf{c a l} / \mathbf{m o l})$} & \multicolumn{3}{c}{$\boldsymbol{\chi}_{\mathbf{1 2}}$} \\
\cline { 2 - 7 } & $\mathbf{2 9 8 , 1 5} \mathbf{~ K}$ & $\mathbf{3 1 3 , 1 5} \mathbf{~ K}$ & $\mathbf{3 2 3 , 1 5 ~ K}$ & $\mathbf{2 9 8 , 1 5} \mathbf{~}$ & $\mathbf{3 1 3 , 1 5} \mathbf{~ K}$ & $\mathbf{3 2 3 , 1 5} \mathbf{~ K}$ \\
\hline 0,027 & 18,41 & 18,39 & 18,38 & 1,375 & 1,320 & 1,285 \\
0,055 & 36,52 & 36,49 & 36,45 & 1,395 & 1,339 & 1,303 \\
0,116 & 71,45 & 71,37 & 71,29 & 1,437 & 1,380 & 1,343 \\
0,183 & 103,77 & 103,62 & 103,47 & 1,484 & 1,425 & 1,387 \\
0,259 & 132,03 & 131,79 & 131,56 & 1,537 & 1,475 & 1,435 \\
0,343 & 154,20 & 153,83 & 153,50 & 1,597 & 1,532 & 1,490 \\
0,440 & 167,31 & 166,79 & 166,35 & 1,665 & 1,597 & 1,553 \\
0,550 & 167,04 & 166,35 & 165,80 & 1,744 & 1,671 & 1,624 \\
0,677 & 146,85 & 146,04 & 145,44 & 1,836 & 1,758 & 1,708 \\
0,825 & 96,63 & 95,91 & 95,40 & 1,947 & 1,862 & 1,807 \\
0,909 & 55,50 & 55,02 & 54,68 & 2,012 & 1,922 & 1,864 \\
\hline
\end{tabular}

\section{Sonuç}

Birçok sanayi dalında yaygın olarak kullanılan etilasetat ve etanolün azeotrop oluşturan ikili karışımının izotermal buharsıvı denge davranışı UNIFAC yöntemi kullanılarak sıvı faz aktivite katsayılarının kestirimi temelinde gerçekleştirilmiştir.

İdeal davranıştan sapmalar genellikle akitivite katsayılarıyla ifade edilirler ve bir karışımda aktivite katsayısı aşırı Gibbs enerjisiyle bağlantılıdır. İdeallikten sapmalar pozitif veya negatif olabilir. Pozitif değerlere sahip bir sistem için ideallikten pozitif sapma olduğu söylenebilir. Tersi durumda ise negatif sapma söz konusudur. Aşırı Gibbs serbest enerjisinin pozitif değerleri birbirine benzemeyen moleküller arasındaki spesifik etkileşimlere işaret eder ve bileşim, sıcaklık ve basıncın bir fonksiyonudur. Ancak en önemli değişken bileşimdir. Raoult yasasından pozitif sapmalar benzer molekülleri arasında güçlü, farklı moleküller arasında ise zayıf kuvvetler olduğunun göstergesidir. Etilasetat-etanol sistemi için görülen pozitif sapmalar ve 1'den büyük aktivite katsayıları moleküller arası hidrojen bağlanması nedeniyle ortaya çıkan etkileşimlerden ve iki bileşen arasındaki çekim kuvvetlerinin zayıf olmasından kaynaklanabilir.

\section{Kaynakça}

Abraham, T., Bery, V., \& Kudchadker, P. (1971). Densities of some substances. Journal of Chemical and Engineering Data, 16(3), 355-356.
Barton, A.F.M. (1975). Solubility parameters. Chemical Reviews, 75, 731-753.

Dean, J.A. (1999). Lange's Handbook of Chemistry, USA: McGraw-Hill.

Fredenslund, A., Jones, R.L., \& Prausnitz, J.M. (1975). Groupcontribution estimation of activity coefficients in nonideal liquid mixtures. AlChE Journal, 21(6), 1086-1099.

Furnas, C.C., \& Leighton, W.B. (1937). Ethyl alcohol-ethyl acetate and acetic acid-ethyl acetate systems. Industrial and Engineering Chemistry, 29(6), 709-710.

Ghanadzadeh, A., Ghanadzadeh, H., \& Alinejad, M. (2012). Dielectric study on polar binary mixtures of (ester-alcohol) at 298.2 K. Iranian Journal of Chemical Engineering, 9(3), 22-30.

Gmehling, J., Rasmussen, P., \& Fredenslund, A. (1982). Vaporliquid equilibria by UNIFAC group contribution. Revision and extension. 2. Industrial \& Engineering Chemistry Process Design Development, 21, 118-127.

Huang, J.-F., \& Lee, L.-S. (1996). Simultaneous estimation of excess enthalpy, excess Gibbs energy and vapor-liquid equilibrium using the modified Wilson model. Fluid Phase Equilibria, 121, 27-43.

Khasanshin, T.S., \& Aleksandrov, A.A. (1984). Thermodynamics properties of ethanol at atmospheric pressure. Journal of Engineering Physics, 47, 1046-1052.

Kobuchi, S., Takakura, K., Yonezawa, S., Fukuchi, K., \& Arai, Y. (2013). Prediction of vapor-liquid equilibria of binary systems containing esters by using Wilson equation with 
parameters estimated from pure-component properties. Fluid Phase Equilibria, 352, 114- 117.

Letyanina, I., Tsvetov, N., Toikka, A. (2016). Application of the UNIFAC models for prediction and description of excess molar enthalpies for binary mixtures of n-propanol, acetic acid, n-propyl acetate, and water. Fluid Phase Equilibria, 427, 202-208.

Mackay, D., Shiu, W.Y., Ma, K.-C., \& Lee, S.C. (2006). Handbook of Physical-Chemical Properties and Environmental Fate for Organic Chemicals. Volume III, $2^{\text {nd }}$ ed., CRC Press, USA: Taylor \&Francis.

Mulder, M.H.V., \& Smolders, C.A. (1986). Pervaporation, solubility aspects of the solution-diffusion model. Separation \& Purification Technology, 15, 1-19.

Mulder, M.H.V., Franken, T., \& Smolders, C.A. (1985). Preferential sorption versus preferential permeability in pervaporation. Journal of Membrane Science, 22, 155-173.

Murti, P.S., \& Van Winkle, M. (1958). Vapor-liquid equilibria for binary systems of methanol, ethyl alcohol, 1-propanol, and 2-propanol with ethyl acetate and 1-propanol-water. Industrial and Engineering Chemistry, 3(1), 72-81.

Nagata, I., Yamada, T., \& Nakagawa, S. (1975). Excess Gibbs free energies and heats of mixing for binary systems ethyl acetate with methanol, ethanol, 1-propanol, and 2-propanol. Journal of Chemical and Engineering Data, 20(3), 271-275.

Nijhius, H.H., Mulder, M.H.V., \& Smolders, C.A. (1993). Selection of elastomeric membranes for the removal of volatile organics from water. Journal of Applied Polymer Science, 47, 2227-2243.

Ortega, J., Pena, J.A., \& de Alfonso, C. (1986). Isobaric vaporliquid equilibria of ethylacetate mixtures at $760 \pm 0.5$ mmHg. Journal of Chemical and Engineering Data, 31, 339-342.

Parsana V.M., \& Parikh S.P. (2015). Vapor-liquid equilibrium data prediction by advanced group contribution methods for a binary system of cyclopentyl methyl ether and acetic acid at atmospheric pressure. Research Journal of Chemical Sciences, 5(6), 64-72.

Pattanaik, B.N., \& Mandalia, H.C. (2011). Ethylacetate: properties, production processes and applications-a review. International Journal of Current Research and Review, 3(12), 23-40.

Poling, B.E., Prausnitz, J.M. \& O'Connell, J.P. (2001). The Properties of Gases and Liquids. NewYork: McGraw-Hill.

Resa, J. M., González, C., Goenaga, J. M., \& Iglesias, M. (2004). Temperature dependence of excess molar volumes of ethanol + water + ethyl acetate. Journal of Solution Chemistry, 33(2), 169-198.

Rhim, J.-W. \& Huang, R.Y.M. (1989). On the prediction of separation factor and permeability in the separation of binary mixtures by pervaporation. Journal of Membrane Science, 46, 335-348.

Ruidiaz, M.A., Vargas, E.F., \& Martínez, F. (2010). Study of some volumetric properties of the pharmaceutical model solvent system ethanol + ethyl acetate at several temperatures. Latin American Journal of Pharmacy, 29(2), 306-312.

Sandler, S.I. (2017). Kimya, Biyokimya ve Mühendislik Termodinamiği. (Çev. Sinan Yapıc1). İzmir: İzmir Güven Kitabevi. (Kitabın Orijinal Basımı 2006).

Susial, P., Sosa-Rosario, A., Rodriguez-Henriquez, J.J., \& RiosSantana, R. (2011). Vapor pressures and VLE data measurements. ethyl acetate+ethanol at $0.1,0.5$ and $0.7 \mathrm{MPa}$ binary system. Journal of Chemical Engineering of Japan, 44(3), 155-163.

Xia, S., Wei, W., Liu, G., Dong, X., \& Jin, W. (2012). Pervaporation properties of polyvinyl alcohol/ceramic composite membrane for separation of ethyl acetate/ethanol/water ternary mixtures. Korean Journal of Chemical Engineering, 29(2), 228-234.

Zhang, D.L., Deng, Y.F., Li, C.B., \& Chen, J. (2008). Separation of ethyl acetate-ethanol azeotropic mixture using hydrophilic ionic liquids. Industrial\& Engineering Chemistry Research, 47, 1995-2001.

Zhang, X.H., Liu, Q.L., Xiong, Y., Zhu, A.M., Chen, Y., \& Zhang, Q.G. (2009). Pervaporation dehydration of ethyl acetate/ethanol/water azeotrope using chitosan/poly (vinyl pyrrolidone) blend membranes. Journal of Membrane Science, 327, 274-280.

Zhang, Z., Wu, K., Zhang, Q., Zhang, T., Zhang, D., Yang, R. \& Li, W. (2017). Separation of ethyl acetate and ethanol azeotrope mixture using dialkylphosphates-based ionic liquids as entrainers. Fluid Phase Equilibria, 454, 91-98. 